\title{
Concerning the depletion width of a radial $p$-n junction and its influence on electrical properties of the diode
}

\author{
V.L. Borblik \\ V. Lashkaryov Institute of Semiconductor Physics, NAS of Ukraine, \\ 41, prospect Nauky, 03680 Kyiv, Ukraine \\ E-mail: borblik@isp.kiev.ua
}

\begin{abstract}
Dependences of the depletion widths in a radial core-shell $p-n$ diode on the radius of metallurgical boundary of the $p-n$ junction have been studied theoretically in detail. While the depletion width of the core increases with decreasing the radius, the depletion width of the shell, on the contrary, decreases. This is the consequence of cylindrical symmetry of the structure. And the total depletion width of the $p$ - $n$ junction can both increase and decrease depending on doping levels of the core and shell. A number of cases is presented where the dependence of depletion width of the $p-n$ junction on its curvature influences on the diode current-voltage characteristics.
\end{abstract}

Keywords: nanostructures, core-shell nanowire, radial $p$ - $n$ junction, depletion width.

Manuscript received 26.01.17; revised version received 05.04.17; accepted for publication 14.06.17; published online 18.07.17.

\section{Introduction}

It has been established that an interface curvature of doped nano-dimensional semiconductor structures enlarges their depletion length. For the samples of cylindrical symmetry, it has been shown in paper [1], and for the case of spherical symmetry - in the papers $[2,3]$. In those papers, it was talked of depleting toward the interior of the sample.

A $p-n$ junction is the interface where depletion occurs on both its sides. This problem (with regard to a radial core-shell $p$ - $n$ diode) has been considered in Ref. 4 where authors have developed a general electrostatic theory for these structures and have marked out four types of electric structure for radial $p-n$ junction diode depending on the set of parameters. However, any explicit dependence of the depletion widths for both sides of the $p-n$ junction on its radius has been not established. Partially this has made in the work [5] but only for the depletion width of the core which (likely to the case of semi-limited structures [1-3]) increases with decreasing radius of metallurgical boundary of the $p-n$ junction. Behavior of the depletion width of the nanowire shell as well as of the whole depletion width has remained not studied.

Nothing of this has been also reported in the papers [6-8] devoted to numerical calculations of radial coreshell $p-n$ junction solar cells. Meanwhile, the dependence of depletion width for the nanowire shell on radius of the $p-n$ junction proves to be completely different. And variation of the total depletion width versus $p$ - $n$ junction radius proves to be non-trivial.

\section{Calculation of the depletion widths}

Let us consider the case of partially depleted $p$-core and $n$-shell (Fig. 1). We will proceed from the known system of 2 equations $[4,5]$, which allow us to determine the depletion widths in the core $w_{p}=r_{0}-r_{p}$ and in the shell $w_{n}=r_{n}-r_{0}$, where $r_{0}$ is the core radius, $r_{p}$ is the depletion region boundary in the core, $r_{n}$ is the depletion region boundary in the shell, and $r_{d}$ is external radius of the nanowire. 


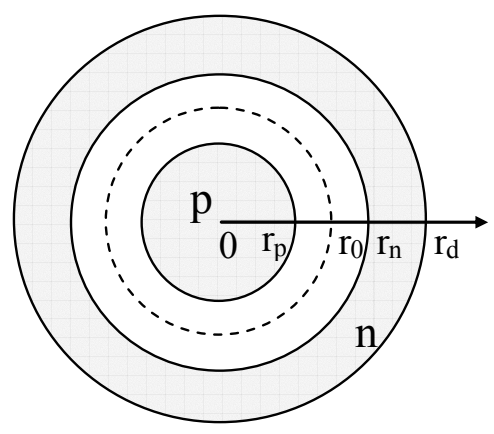

Fig. 1. Schematic view of the radial $p-n$ structure.

From matching the electric fields in the point $r_{0}$, we have

$N_{A}\left(r_{0}^{2}-r_{p}^{2}\right)=N_{D}\left(r_{n}^{2}-r_{0}^{2}\right)$

( $N_{A}$ and $N_{D}$ are the concentrations of acceptors and donors, respectively) and matching the potentials in the same point gives

$\frac{q}{2 \varepsilon_{s}}\left(N_{A} r_{p}^{2} \ln \frac{r_{p}}{r_{0}}+N_{D} r_{n}^{2} \ln \frac{r_{n}}{r_{0}}\right)=V_{b i}$,

where $q$ is the electron charge, $\varepsilon_{s}$ - dielectric constant of the semiconductor, $V_{b i}$ - built-in potential of the $p-n$ junction [9]

$V_{b i}=\frac{k T}{q} \ln \frac{N_{A} N_{D}}{n_{i}^{2}}$,

$k$ is the Boltzmann constant, $T$ - temperature, $n_{i}$-concentration of intrinsic carriers. Expressing $r_{n}$ in terms of $r_{p}$

$r_{n}=\sqrt{r_{0}^{2}+\left(r_{0}^{2}-r_{p}^{2}\right) N_{D} / N_{A}}$,

one can obtain the following transcendental equation in $r_{p}$ :

$$
\begin{aligned}
& \left(1+\frac{N_{A}}{N_{D}}\left(1-\frac{r_{p}^{2}}{r_{0}^{2}}\right)\right) \ln \sqrt{1+\frac{N_{A}}{N_{D}}\left(1-\frac{r_{p}^{2}}{r_{0}^{2}}\right)}+ \\
& +\frac{N_{A}}{N_{D}} \frac{r_{p}^{2}}{r_{0}^{2}} \ln \frac{r_{p}}{r_{0}}-\frac{V_{b i} 2 \varepsilon_{s}}{q N_{D} r_{0}^{2}}=0 .
\end{aligned}
$$

For numerical solution of Eq. (5), the parameters of silicon at room temperature were chosen as follows: $\varepsilon_{s}=12 \varepsilon_{0} \quad\left(\varepsilon_{0}\right.$ is the permittivity of free space $), n_{i}=$ $6.3 \cdot 10^{9} \mathrm{~cm}^{-3}$. The calculation results are presented in Figs. 2a-c. Fig. 2a corresponds to the case when the core is doped higher than the shell $\left(N_{A}=5 \cdot 10^{18} \mathrm{~cm}^{-3}\right.$, $N_{D}=5 \cdot 10^{17} \mathrm{~cm}^{-3}$ ), Fig. $2 \mathrm{~b}$ concerns the opposite case $\left(N_{A}=5 \cdot 10^{17} \mathrm{~cm}^{-3}, \quad N_{D}=5 \cdot 10^{18} \mathrm{~cm}^{-3}\right), \quad$ and Fig. $2 \mathrm{c}$ represents the results for the case of equal doping levels $\left(N_{A}=N_{D}\right)$.
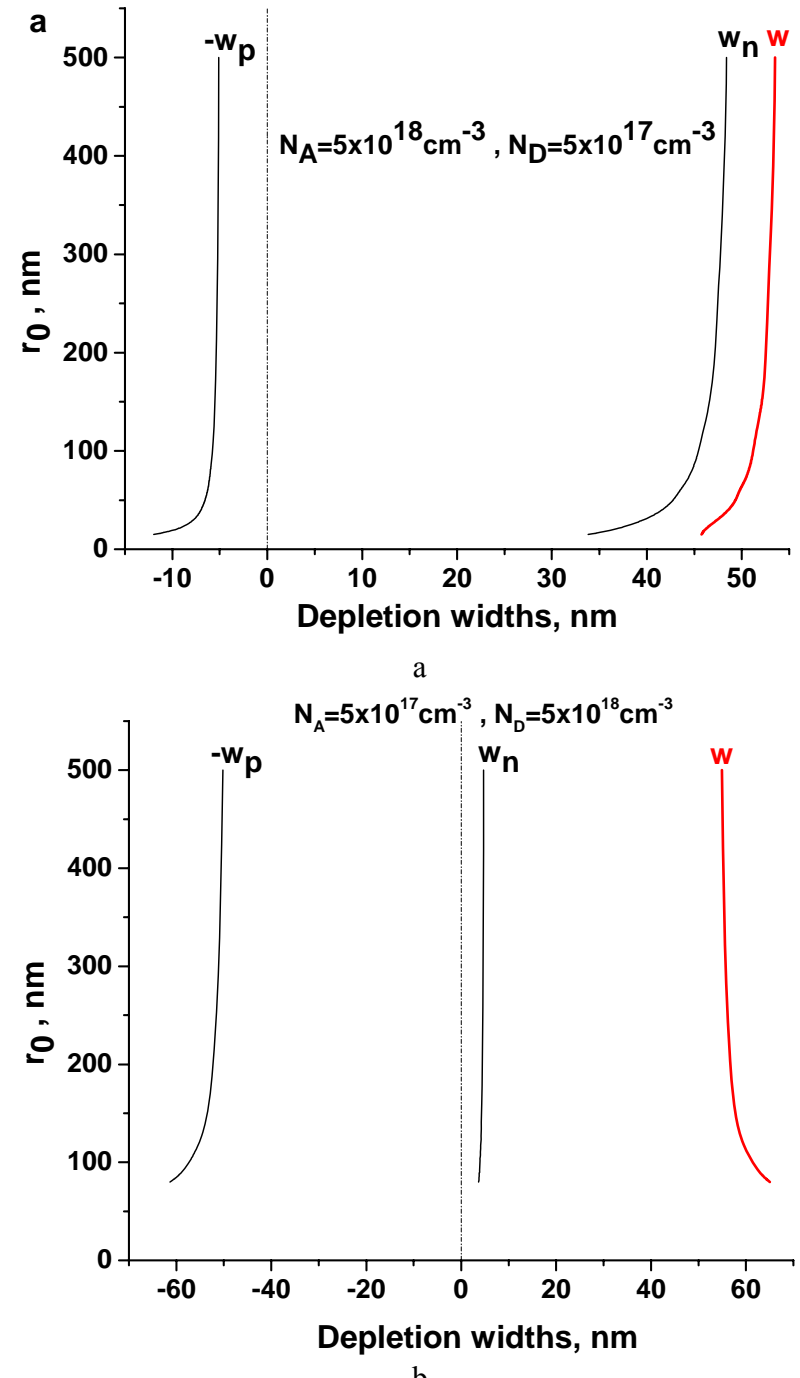

b

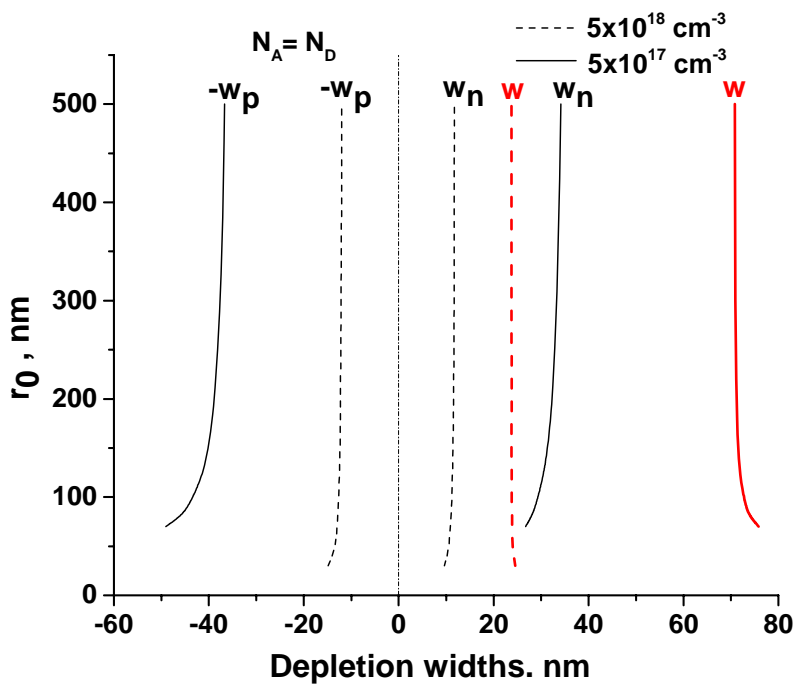

Fig. 2. Dependence of the depletion widths in the core $w_{p}$, in the shell $w_{n}$, and the total depletion width $w$ on radius of the $p$ $n$ junction at $N_{A} \gg N_{D}(a), N_{A}<<N_{D}(b)$ and $N_{A}=N_{D}(c)$ (abscissa equal to zero corresponds to metallurgical boundary of the $p-n$ junction). 
As it follows from these figures, in all three cases the depletion width of the core increases with decreasing its radius, meanwhile, the depletion width of the shell, on the contrary, decreases. As for the whole depletion width of the $p-n$ junction $w=w_{p}+w_{n}$, it can both increase and decrease and even be nearly independent of the $p$ - $n$ junction radius at equal and sufficiently high doping levels of both its sides.

Opposite character of dependencies on the $p-n$ junction radius of the depletion widths for the core and shell is a consequence of radial falling that is characteristic feature for solutions of differential equations in the cylindrical (as well as spherical) coordinate system. In particular, this fact has shown itself in the paper [10] where solution of the discontinuity equation in the core-shell nanowire demonstrates an accelerated decay of the nonequilibrium carrier concentration in the shell and its slowed down decay in the core.

Here, analogous situation is available. The built-in electric field of the $p-n$ junction, which is maximal at its metallurgical boundary, decreases in direction of the shell faster than in direction of the core. It is for this reason that at equal doping levels in $p$ - and $n$-sides, the region of electric field in the shell proves to be always shorter than in the core (as it has been found in [5]).

Note also that the "mysterious" increase in depletion length in semi-limited semiconductor nanostructures with cylindrical and spherical geometry (as in [1-3]) has the same nature.

\section{The current-voltage characteristics}

Under biasing the diode by the voltage $U$, the total depletion width changes its dimensions: it narrows under the forward voltage and expands under the reverse voltage. This means that the points where injection of nonequilibrium carriers takes place, i.e. $r_{p}$ and $r_{n}$, drift to the region of different curvature of the $p-n$ junction. And this fact, as it has been shown in [10], varies the current density at the expense of the curvature additionally to the applied voltage.

In accordance with [10], the diode current density from the core to the shell is where $\quad J_{p 0}=q \sqrt{D_{p} / \tau_{p}} \Delta p_{0}\left(x_{n}\right)(\exp (q U / k T-1)$, $x_{n}=r_{n} / L_{p}, x_{d}=r_{d} / L_{p}, L_{p}=\sqrt{D_{p} \tau_{p}}, D_{p}$ and $\tau_{p}$ are the diffusion coefficient and the lifetime of nonequilibrium holes in $n$-shell, $I_{0}$ and $K_{0}$ are modified Bessel's functions of the $1^{\text {st }}$ and $2^{\text {nd }}$ kinds, respectively, $\Delta p_{0}\left(x_{n}\right)$ is the equilibrium hole concentration at the depletion region boundary in the $n$-shell (emitter of holes), $S^{*}=S L_{p} / D_{p}-$ dimensionless surface recombination velocity at the external contact to the shell.

In the case of planar diode, the corresponding current density takes the form

$$
{ }_{p^{+}-n}^{p l}=J_{p 0} \frac{\left(1+S^{*}\right) e^{x_{d}-x_{n}}-\left(1-S^{*}\right) e^{-\left(x_{d}-x_{n}\right)}}{\left(1+S^{*}\right) e^{x_{d}-x_{n}}+\left(1-S^{*}\right) e^{-\left(x_{d}-x_{n}\right)}} .
$$

The ratio $J_{p^{+}-n} / J_{p^{+}-n}^{p l}$ at the same parameters $x_{n}, x_{d}$, and $S^{*}$ may be called as the enhancement coefficient for the current density at the expense of the diode curvature.

Under the forward bias, when the diode current varies exponentially with the applied voltage, the influence of this factor reduces to practically equal current rise in all the range of applied voltages. But under the reverse bias, variation of the enhancement coefficient at the expense of the varying curvature can result in qualitatively new effects.

Fig. 3 represents the reverse current-voltage characteristics of the radial diode $\left(\right.$ at $S^{*}=0$ and $\left.S^{*}=\infty\right)$ in comparison with those of planar diode at the same values of parameters. It is the variation of the enhancement coefficients, connected with the curvature variation (shown in the inserts), that results in decreasing current density with the reverse bias.

Fig. 4 presents calculation results for another case, when the boundary of depletion region in the shell comes nearer and nearer to the external contact $r_{d}$ with increasing the reverse voltage. In the radial diode, this approach occurs at appreciably higher voltage values than in the planar diode with the same parameter values due to significant difference in the depletion region widths.

$$
J_{p^{+}-n}=J_{p 0} \frac{K_{1}\left(x_{n}\right)\left[I_{1}\left(x_{d}\right)+S^{*} I_{0}\left(x_{d}\right)\right]-I_{1}\left(x_{n}\right)\left[K_{1}\left(x_{d}\right)-S^{*} K_{0}\left(x_{d}\right)\right]}{I_{0}\left(x_{n}\right)\left[K_{1}\left(x_{d}\right)-S^{*} K_{0}\left(x_{d}\right)\right]+K_{0}\left(x_{n}\right)\left[I_{1}\left(x_{d}\right)+S^{*} I_{0}\left(x_{d}\right)\right]}
$$



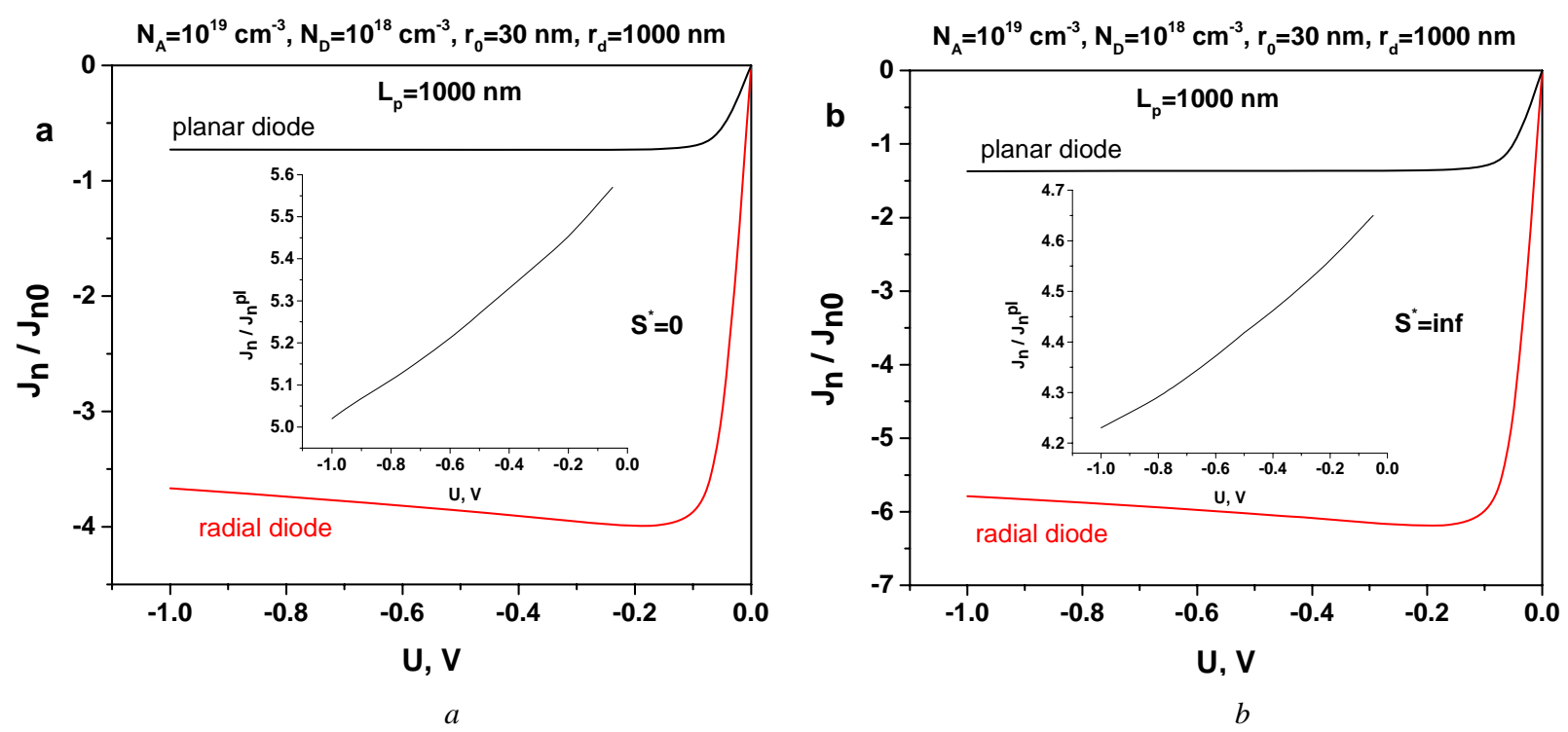

Fig. 3. Reverse current-voltage characteristics of the radial diode at $\mathrm{S}^{*}=0(a)$ and $\mathrm{S}^{*}=\infty(b)$ under conditions when the curvature varies with applied voltage, in comparison with those of planar diode at the same values of the parameters; the inserts shows dependences of the enhancement coefficients for the current density on the voltage.
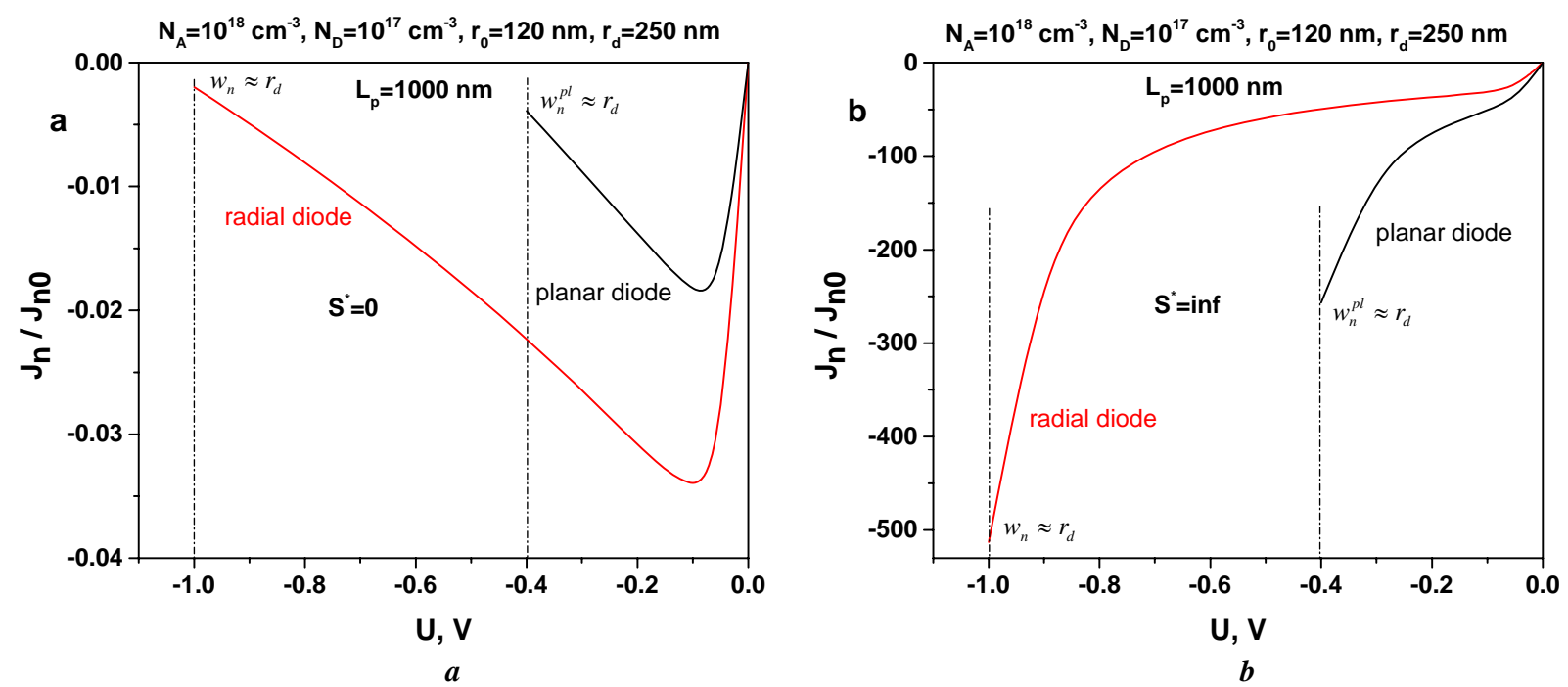

Fig. 4. Reverse current-voltage characteristics of the radial diode at $S^{*}=0(a)$ and $S^{*}=\infty(b)$ under conditions when the depletion region almost approaches the external boundary of the nanowire, in comparison with those of planar diode at the same values of the parameters.

\section{Conclusions}

Thus, in radial diodes dependences of the depletion widths in the core and shell on the $p-n$ junction radius have opposite character due to cylindrical symmetry of the nanowire. And dependence of the total depletion width on the $p$ - $n$ junction radius proves to be ambiguous and depending on the doping levels of the core and shell. Furthermore, the $p-n$ junction curvature can result in not only quantitative but also qualitative effects in the diode current-voltage characteristics.
It is worth to note also that since under conditions of high doping, the total depletion width of the radial diode depends on the $p$ - $n$ junction curvature rather weakly, inter-band tunneling probability remains nearly the same as in planar diode. This means that the excess tunnel current remains practically the same. For the same reason, the $p$ - $n$ junction curvature does not influence essentially on performance of tunnel core-shell diodes. In particular, this fact is corroborated by successful fabrication of a tunnel GaAs core-shell diode [11]. 


\section{Acknowledgement}

This work was supported by the National Academy of Sciences of Ukraine [project 2.2.6.34].

\section{References}

1. Luscombe J.H., Frenzen C.L. Depletion lengths in semiconductor nanostructures. Solid State Electron. 2002. 46, No. 6. P. 885-889.

2. Nersesyan S.R., Petrosyan S.G. Depletion length and space charge layer capacitance in doped semiconductor nanosphere. Semicond. Sci. Technol. 2012. 27, No. 12. P. 125009.

3. Borblik V.L. Depletion length in semiconductor nanostructures with spherical symmetry. SolidState Electron. 2015. 114. P. 171-173.

4. Chia A.C.E., LaPierre R.R. Electrostatic model of radial $p$ - $n$ junction nanowires. J. Appl. Phys. 2013. 114, No. 7. P. 074317.

5. Petrosyan S., Yesayan A., Nersesyan S. Theory of nanowire radial p-n-junction. World Acad. Sci. Eng. Technol. 2012. 71. P. 1065-1070.
6. Kayes B.M., Atwater H.A., Lewis N.S. Comparison of the device physics principles of planar and radial p-n junction nanorod solar cells. J. Appl. Phys. 2005. 97, No. 11. P. 114302.

7. Ali N.M., Allam N.K., Haleem A.M.A., Rafat N.H. Analytical modeling of the radial $p-n$ junction nanowire solar cells. J. Appl. Phys. 2014. 116, No. 2. P. 024308 .

8. Ali N.M., Haleem A.M.A., Allam N.K., Rafat N.H. Numerical simulation and a parametric study of inorganic nanowire solar cells. Int. J. Numer. Model. 2017. 30. P. e2176.

9. Sze S.M., Ng K.K. Physics of Semiconductor Devices, 3rd ed. John Wiley \& Sons, 2007.

10. Borblik V. Effect of circular p-n junction curvature on the diode current density. J. Electron. Mater. 2016. 45, No. 8. P. 4117-4121.

11. Darbandi A., Kavanagh K.L., Watkins S.P. Lithography-free fabrication of core-shell $\mathrm{GaAs}$ nanowire tunnel diodes. Nano Lett. 2015. 15, No. 8. P. 5408-5413. 